\title{
Criminologie
}

\section{Les gangs et la violence dans leur quartier tels que perçus par les jeunes}

\section{Gangs and Gang Violence : What Non-Gang Member} Adolescents Think about Gangs and Gang Violence in their Neighborhood

\section{Las pandillas callejeras y la violencia en sus barrios. Percepciones de jóvenes que no son miembros de las pandillas}

\section{Sarah Kelly}

Volume 48, numéro 2, automne 2015

Nouvelles perspectives sur le phénomène des gangs de rue

URI : https://id.erudit.org/iderudit/1033840ar

DOI : https://doi.org/10.7202/1033840ar

Aller au sommaire du numéro

Éditeur(s)

Les Presses de l’Université de Montréal

ISSN

0316-0041 (imprimé)

1492-1367 (numérique)

Découvrir la revue

Citer cet article

Kelly, S. (2015). Les gangs et la violence dans leur quartier tels que perçus par les jeunes. Criminologie, 48(2), 125-138. https://doi.org/10.7202/1033840ar

\section{Résumé de l'article}

La violence des gangs est un problème de société en pleine croissance. La présente étude a pour but de se pencher sur la perception qu'ont les adolescents, non membres de gangs, à propos des gangs, de la violence qui y est associée et de l'influence qu'elle exerce, selon eux, sur leur quartier. Vingt adolescents ont participé à des groupes de discussion sous forme d'entretiens semi-dirigés, ce qui a permis de dégager quatre thèmes principaux : 1) un sentiment d'appartenance ;2) un prix à payer ; 3) l'incompréhension ; et 4) l'évitement actif. Les perceptions à propos des gangs et de la violence diffèrent d'un adolescent à l'autre. Comprendre leurs points de vue pourrait aider à la conception de programmes de santé publique visant à éduquer les jeunes sur les gangs et sur les conséquences d'une implication dans de tels groupes. 


\title{
Les gangs et la violence dans leur quartier tels que perçus par les jeunes
}

\author{
Sarah Kelly, Ph. D., inf. aut. ${ }^{1}$ \\ Professeure adjointe, École des sciences infirmières \\ Rutgers, Université d'État du New Jersey \\ Sarah.kelly@rutgers.edu
}

\section{Article traduit de l'anglais par Florence Dubois}

RÉSUMÉ - La violence des gangs est un problème de société en pleine croissance. La présente étude a pour but de se pencher sur la perception qu'ont les adolescents, non membres de gangs, à propos des gangs, de la violence qui y est associée et de l'influence $q u$ 'elle exerce, selon eux, sur leur quartier. Vingt adolescents ont participé à des groupes de discussion sous forme d'entretiens semi-dirigés, ce qui a permis de dégager quatre thèmes principaux: 1) un sentiment d'appartenance; 2) un prix à payer; 3) l'incompréhension; et 4) l'évitement actif. Les perceptions à propos des gangs et de la violence diffèrent d'un adolescent à l'autre. Comprendre leurs points de vue pourrait aider à la conception de programmes de santé publique visant à éduquer les jeunes sur les gangs et sur les conséquences d'une implication dans de tels groupes.

MOTS CLÉS • Jeunes, gangs, violence des gangs, quartier, communauté.

Selon le modèle écologique de développement humain de Bronfenbrenner (1979), les adolescents sont directement influencés par leur environnement social ou physique et indirectement influencés par les contextes culturel et historique dans lesquels ils vivent. Ils apprennent de cet environnement (Bronfenbrenner, 1979). Des recherches ont pu montrer que plusieurs facteurs environnementaux influencent la perception qu'ont les jeunes à propos des gangs de rue, notamment les interactions sociales avec leurs pairs et leurs relations familiales (Thornberry, Krohn,

1. Rutgers University, SSB, 11th Floor, Room 1126, 65 Bergen Street, Newark (New Jersey), 07101, États-Unis. 
Lizotte, Smith et Tobin, 2003). Ces facteurs agiraient à des degrés variables sur leur vision des gangs de rue, des membres de ces groupes et des effets de leur présence sur la sécurité de leur quartier (WalkerBarnes et Mason, 2001).

En fait, c'est auprès des membres de leur famille et de leur communauté que les adolescents apprennent sur le sujet. L'information ainsi obtenue peut toutefois être imprécise ou éloignée de la réalité des membres des gangs et des raisons qui les poussent à la violence. Les jeunes peuvent également obtenir un portrait irréaliste des gangs, véhiculé par la télévision et le cinéma. Dans la société, on représente généralement à tort leurs membres comme des êtres méprisables, alors qu'ils ne cherchent qu'à faire partie de cette société (Klein et Maxson, 2006). La première étape en vue de sensibiliser les adolescents à risque de se joindre aux gangs de rue consiste donc à comprendre la manière dont ils perçoivent les gangs et la violence. La présente étude vise ainsi à analyser la perception des adolescents, non membres de gangs, à propos des gangs et de la violence qui y est associée.

\section{Comment les jeunes perçoivent-ils les gangs de rue?}

De nombreux adolescents sont exposés à la présence des gangs et à leur violence (Kelly, Anderson et Peden, 2009). Mais bien que ces bandes soient actives dans leur quartier, ce serait les interactions avec les pairs et la famille qui influenceraient le plus la perception et la participation des jeunes à ces groupes (Dishion, Nelson et Yasui, 2005; Kelly et Anderson, 2012). Walker-Barnes et Mason (2001) ont relevé, par exemple, qu'il y avait un lien entre une faible activité des gangs et la capacité des parents à offrir un milieu stable et réconfortant à leurs enfants. Selon Thornberry et al. (2003), les jeunes Afro-Américains, les adolescents dont les parents sont peu éduqués et ceux qui vivent en famille monoparentale seraient plus portés à percevoir les gangs comme une famille et à participer à leurs activités pour s'y sentir acceptés. Les mêmes recherches ont également permis de mettre en évidence que les garçons adolescents étaient plus susceptibles de se joindre à un gang s'ils recevaient peu de soutien de la part de leurs parents. La dyade parentsadolescents jouerait un rôle important, à la fois dans la perception qu'ont les jeunes non membres de gangs à propos de ces groupes, dans leur désir de s'y impliquer ou non, et dans l'influence que la violence de ces groupes aura sur leur développement (Kelly et Anderson, 2012). 
Les pairs, eux aussi, participent largement à forger l'opinion des adolescents sur les gangs de rue (Kelly et Anderson, 2012; Thornberry et al., 2003). Les jeunes garçons ont en effet plus tendance à prendre part aux activités de gangs lorsque leurs camarades versent dans la délinquance et adoptent des comportements sexuels à risque (Thornberry et al., 2003; Walker-Barnes et Mason, 2001). En fait, une forte activité délinquante chez les pairs serait directement associée à leur appartenance à des gangs. Cette corrélation semble toutefois diminuer au fil des années (Thornberry et al., 2003). Par ailleurs, les études de Dishion et al. (2005) indiqueraient que le caractère antisocial de certains adolescents les prédisposerait à se joindre à un gang de rue. Les médias, la famille et les pairs contribueraient ainsi à influencer l'opinion qu'ont les adolescents non membres de gangs sur ceux-ci et sur les violences qui sont liées à leurs activités. Mais il demeure qu'une grande part des adolescents ayant participé aux recherches qui ont été menées sur le sujet sont des membres actifs de gangs de rue.

\section{Méthodologie}

\section{Conception de l'étude et échantillon}

Pour tenter de comprendre la perception des adolescents non membres à l'égard du phénomène des gangs et des violences qui lui sont associées, l'étude a privilégié les groupes de discussion. Les jeunes sélectionnés venaient de quatre centres communautaires, situés dans des quartiers d'une ville aux États-Unis où la violence est présente. L'étude a reçu l'approbation du comité d'examen de l'établissement et du Service des loisirs et des parcs. Des dépliants d'information ont été déposés dans les centres communautaires, et les chercheurs ont participé à de nombreuses activités ciblant les jeunes en vue de distribuer eux-mêmes de l'information aux parents. Des dépliants ont été également distribués aux adolescents à leur sortie des centres afin qu'ils les remettent à leurs parents. Les chercheurs ont ensuite pris des dispositions auprès des parents des jeunes souhaitant participer à l'étude à propos des rencontres, de l'objet de l'étude et du consentement parental.

Vingt-cinq jeunes ont ainsi participé à des rencontres, en groupes de discussion, ayant eu lieu dans les centres communautaires. Selon les critères de l'étude, les jeunes se situent tous dans la tranche d'âge des 13 à 17 ans et de la $1^{\text {re }}$ à la $5^{\mathrm{e}}$ année du secondaire. Ils vivent dans des 
quartiers marqués par la présence des gangs et par une activité criminelle élevée (selon les rapports des forces de l'ordre). Les adolescents devaient de plus participer à l'étude volontairement et avec l'accord de leurs parents. Ont été exclus de cette sélection les adolescents ne parlant ni ne lisant l'anglais, ceux dont les parents ne parlent ni ne lisent l'anglais, les membres de gangs, les pupilles de l'État, les jeunes placés en famille d'accueil et enfin, ceux dont l'état de santé les empêchait de participer à l'étude. Tous les participants ont reçu une carte-cadeau de 10 dollars de Walmart.

\section{Entretiens}

Les participants aux groupes de discussion ont répondu aux questions dans le cadre d'un entretien semi-dirigé conçu par la chercheure en vue d'obtenir leur point de vue sur les gangs et les violences qui y sont reliées. Les entretiens comportaient huit questions, générales et spécifiques, telles que: «Pourquoi pensez-vous que les jeunes se joignent à des gangs?», «Pourquoi, selon vous, ces gangs s'engagent-ils dans des actes violents?», «Comment vivez-vous cette réalité dans votre quartier?». Ces questions servaient donc à aller chercher de l'information détaillée et à pousser le raisonnement des participants. Des données démographiques avaient été préalablement collectées auprès de chacun d'eux.

Quatre groupes de discussion ont donc été réalisés dans des salles privées de chaque centre communautaire dans le but de minimiser d'éventuelles distractions. Pour des raisons d'efficacité, des règles de base ont été établies dans les groupes de discussion avant la collecte des données. Chaque entretien, d'environ 45 minutes, a été enregistré en fichier audio et des notes ont été prises par l'assistant de recherche. Chaque groupe de discussion était composé de cinq répondants, garçons et filles, soit le nombre idéal de participants pour une étude menée auprès d'adolescents.

\section{Analyse des données}

Les données de chaque groupe de discussion ont été transcrites textuellement par un audiotypiste professionnel en un texte écrit dont l'exactitude a ensuite été vérifiée en le comparant au contenu audio. Les notes ont été ajoutées à la version écrite. La chercheure et un chercheur 
qualitatif chevronné ont ensuite analysé le tout selon la méthode de Krueger et Casey (2000), en prenant en compte des facteurs d'occurrence, de spécificité, d'émotivité et d'étendue. La gestion et l'analyse des données ont été effectuées à l'aide du logiciel Atlas Ti (Berlin, Allemagne, 2003) et les codes obtenus ont été regroupés par thèmes (Creswell, 1998). À la suite de l'analyse, la fiabilité des données a été vérifiée grâce à diverses méthodes telles que la récapitulation entre cochercheurs et la vérification des répondants (des adolescents ayant participé à la recherche). Le chercheur qualitatif a de plus vérifié puis validé les données et les codes obtenus.

\section{Constats}

\section{Caractéristiques de l'échantillon}

Vingt adolescents de 13 à 16 ans ( $\mathrm{n}=11$ garçons et $\mathrm{n}=9$ filles) ont participé aux quatre groupes de discussion. La majorité d'entre eux était d'origine afro-américaine $(80 \%)$ et venait de milieux familiaux variés. Plusieurs avaient de nombreux demi-frères et demi-sœurs, sans vivre sous le même toit qu'eux. De plus, plusieurs jeunes vivaient avec un seul parent ou un tuteur légal dans des logements sociaux. Quelques adolescents $(20 \%)$ avaient un parent ayant purgé une peine de prison. Tous fréquentaient l'école secondaire publique $\left(1^{\text {re }}\right.$ à la $5^{\mathrm{e}}$ année du secondaire) et participaient à des activités parascolaires (équipes de basketball ou de meneuses de claques) ou à des activités organisées par les centres communautaires. Cette étude portait sur ces jeunes parce qu'ils vivent dans des milieux où les gangs de rue sont très présents sans eux-mêmes succomber à ce style de vie délinquant. Ces adolescents se sont vu attribuer des pseudonymes pour des raisons de confidentialité.

Pour des raisons inconnues, cinq adolescents se sont abstenus de participer à l'étude. Même si ces jeunes s'étaient portés volontaires et avaient obtenu la permission de leurs parents, ils ne se sont toutefois pas présentés à la séance de discussion. Le même jour où trois d'entre eux devaient participer à un groupe de discussion, un jeune de 16 ans s'est fait tirer dans un immeuble résidentiel non loin du centre communautaire où se déroulait l'entrevue. Cet incident a sans doute influencé la participation de ces jeunes.

L'analyse des données a permis de dégager quatre thèmes principaux : 1) un sentiment d'appartenance; 2) un prix à payer ; 3) l'incompréhension; et 4) l'évitement actif. Ces thèmes se subdivisent en 
plusieurs sous-thèmes tels que la protection, l'inclusion et le soutien moral, dans le cas du sentiment d'appartenance.

\section{Un sentiment d'appartenance}

Treize adolescents ont évoqué le fait que les jeunes entrent dans des gangs parce qu'ils n'ont personne d'autre vers qui se tourner. Le jeune Jeff résume ce sentiment par l'affirmation suivante:

La plupart des gens entrent dans les gangs parce que, genre, comment ils ont grandi, genre, dans des familles d'accueil et tout, ou si t'as des parents qui boivent ou te battent ou prennent de la drogue, ils pensent que les gangs les aiment, qu'elle est leur famille.

Les notions de soutien et de protection ont été souvent évoquées durant les discussions de groupe. Plusieurs adolescents ont tenu des propos tels que: "Quand tu es dans un gang, tu peux toujours compter sur eux, sur les gens du gang. T'es genre, on va faire ça, et ça, alors ils sont genre, ouais, ils ont un groupe d'amis qui les entourent pour qu'ils soient en sécurité et tout.» D'autres mentionnaient que «c'est une protection» ou «quand les choses tournent au vinaigre, ils te laissent pas tomber». Ces adolescents soutenaient que leurs pairs se joignent à des gangs de rue parce que ces groupes génèrent un sentiment d'appartenance qu'ils ne trouvent pas à la maison. La protection, l'inclusion et le soutien moral offerts dans les gangs sont donc l'attrait principal pour ce mode de vie.

Malgré ces affirmations, les jeunes reconnaissent également des aspects négatifs à ce mode de vie, une opinion défendue par Betty dans l'affirmation suivante:

Ils te donnent un endroit où dormir et s'occupent de toi, mais il y a quand même des risques parce que, si tu te fais prendre, mais que t'as rien fait, genre une fusillade à laquelle ton gang participe, mais tu fais rien, tu es dans le gang donc tu dois quand même assumer la responsabilité pour un membre de ta famille. Et vraiment, un gang, c'est la famille de quelqu'un, mais ça dépend de quel genre de gang c'est. Parce qu'il peut être ta famille, mais ta famille peut faire des choses mauvaises.

Parmi les adolescents de l'étude, les perceptions à propos des gangs de rue variaient, mais la majorité d'entre eux sentaient fortement que les jeunes qui s'impliquent dans des gangs sont en quête d'une structure familiale qui manque à leur vie, même si un tel environnement est teinté de violence. 


\section{Un prix à payer}

Selon les jeunes ayant participé à l'étude, pour vouloir être dans un gang, y appartenir et participer à ses activités violentes, il y aurait un prix à payer qui serait parfois élevé. Plusieurs adolescents $(n=6)$ ont nommé le vol et l'agression d'une tierce personne, voire le meurtre, comme les rites de passage à réaliser afin d'entrer dans un gang. Par exemple, selon John: "Il faut que tu voles quelqu'un ou que tu tues quelqu'un juste pour entrer dans le groupe.» Selon Bobby, pour faire partie d'un gang: «Oh, il faut battre [la personne] ou [1a] tuer.» Pourtant, Kathy explique: «La majorité des gens décident juste de se battre, parce que c'est juste se battre et tes blessures vont guérir.»

Infliger des souffrances à d'autres ne serait pourtant pas le seul prix à payer pour se faire accepter. Quelques adolescents $(n=5)$ ont mentionné que plusieurs entrent dans des bandes par un "passage à tabac», pour les garçons, ou en ayant des relations sexuelles avec au moins un des membres, pour les filles. Selon la description de Mike: «Ils les emmènent aux toilettes et ils [les] tabassent... parce qu'ils sont tous dans des classes différentes, et là, ils se font battre dans les toilettes.» De plus, il a été relevé que «si tu es une fille, il faut que tu [couches] avec tout le monde dans le groupe».

Deux jeunes, Tim et Jeff, ont aussi suggéré qu'il faut, pour monter dans la hiérarchie, que certains membres aient recours à des actes violents dans l'espoir qu'ils soient remarqués par leur gang et perçus comme le prix qu'ils payent pour cette reconnaissance. Selon Tim, «si tu te bas, si tu te bas avec quelqu'un, tu montes de rang». Il a également été dit que «s'ils le font [ces actes de violence], c'est pour atteindre un statut plus important [dans le gang], pour montrer qu'ils sont forts». Au dire de ces adolescents, vouloir faire partie d'un gang ou y être associé mène à s'engager dans des actes violents ou à en être victime, un prix trop élevé à payer pour ce désir d'appartenance.

\section{L'incompréhension}

Dix adolescents ont témoigné de leur incompréhension totale quant à la raison pour laquelle des jeunes entrent dans des gangs et y perpétuent des actes violents. Ils voient là un comportement sans but qui peut mener à des situations fâcheuses, parfois à se blesser. 


\section{Susan explique:}

Je crois qu'il ne devrait pas y avoir de gangs, ça devrait juste être un petit groupe avec qui tu peux traîner. Il ne devrait pas y avoir de drame ni rien. Tu devrais pouvoir aller où tu veux sans que personne te dise rien ou qui, ou plus ou moins qui tu dois être ou quel genre de choses ils peuvent faire. Ça n'a aucun sens ni aucun but. Et genre, quand ils se saluent entre eux, il faut toujours qu'ils aient une poignée de main de cinq heures, ça suffit, tu peux juste les saluer et passer à autre chose.

Un autre jeune dit encore: "Pour être honnête, je ne les aime vraiment pas parce qu'il faut subir tellement de choses pour pouvoir en faire partie, et moi je dis que c'est une perte de temps. Je veux dire, tu peux juste être toi-même.»

Joe, quant à lui, soutient:

Je n'aime pas les gangs parce que c'est stupide, parce que tu ne vas jamais assumer ta couleur. Ça sera pas rouge, ça sera jamais bleu, alors pourquoi tu te bas et tu tires des gens, tu les tues pour ces couleurs. Et tu meurs, tu meurs. C'est pas toi, les membres de ta gang vont te faire des funérailles et tu vas leur manquer, mais au bout de quelques mois, ils ne pensent plus à toi et ils passent à autre chose. Toute ta famille va te pleurer. C'est pour ça que je ne comprends pas...

Pour plusieurs raisons, ces jeunes avaient le sentiment net qu'être dans un gang serait une perte de temps et mènerait à des expériences de vie négatives. L'idée qu'il faut être dans un gang pour mener une vie remplie d'activités et socialiser les rendait perplexes. Ces adolescents voient le monde autrement et ne comprennent pas pourquoi certains jeunes sont attirés par les gangs.

\section{L'évitement actif}

L'évitement actif des gangs et de leurs activités dans leur quartier est un des principaux sujets mentionnés par les jeunes. Mike a commenté, dans un des groupes: «Je ne veux pas entrer dans un gang, et c'est pour ça que j'ai arrêté de me tenir avec des gens qui sont dans des gangs. Ils vont te dire ceci, ils vont te dire cela, ils vont te ramener les drames de quelqu'un.» Dans l'esprit de ces adolescents, socialiser avec des membres de gangs peut être à la fois dangereux et malsain. Une jeune, Mélissa, a expliqué quant à elle qu'elle vit dans un quartier dangereux et qu'éviter certains endroits est la meilleure manière de rester saine et sauve. Elle ajoute: 
D'habitude, j'appelle ma mère pour qu'elle sache qu'on est bien arrivés. Mon quartier, ce n'est pas le plus sécuritaire, mais c'est mieux que rien. Mais j'habite à côté d'un vendeur de drogue, alors ma vie est en danger tous les jours... Quand je vous dis que je ne sors pas de la maison, vous me verrez jamais dehors, à part quand je vais quelque part, ou que je sors les poubelles, ou que je ramasse le courrier. Je ne m'assois jamais dehors et je ne fais jamais la conversation aux gens parce que j'évite ce genre de choses. Et j'ai beaucoup trop de plans dans la vie pour me mêler à de mauvaises fréquentations. Alors je reste dans la maison.

Ce témoignage montre à quel point les gangs et la violence peuvent influencer la perception qu'ont les jeunes de leur environnement, et pourquoi leurs camarades et eux-mêmes s'abstiennent de participer à des activités à l'extérieur.

Ces jeunes cherchent à éviter les gangs pour se soustraire à leur influence négative et plusieurs d'entre eux $(n=6)$ pensent que rester chez eux le leur permet. Par exemple, Kathy affirme: «Je peux sortir, c'est juste que je ne préfère pas.» Mike, quant à lui, explique: «Si vous voulez vous rendre à l'avenue Phillips, par exemple, vous ne voudriez même pas, genre, mettre les pieds dans ce quartier, vous ne voudriez jamais entrer dans un magasin de ce coin de la ville à cause du milieu.» Selon deux autres jeunes, les gangs et leurs activités «en font un mauvais quartier» et mènent les gens à «le considérer comme un endroit dangereux pour leurs enfants, où ils ne veulent pas les voir traîner». Les jeunes ont donc recours à l'évitement actif afin de se sentir en sécurité dans un milieu dangereux.

\section{Discussion}

La présente étude a analysé la perception qu'ont les adolescents, non membres de gangs, à propos de ces groupes et de la violence qui y est liée. Dans les groupes de discussion, chaque adolescent a exprimé son opinion à l'égard des gangs, des raisons que peuvent avoir les membres de gangs d'agir violemment et de l'influence de ces groupes dans leur quartier. Les jeunes ont apporté des points de vue variés.

Il y a plusieurs raisons pour lesquelles des adolescents se joindraient à un gang et y commettraient des actes violents. Mais nombreux sont les adolescents qui, avec du recul, considèrent cette idée comme absurde, voire comme une perte de temps colossale. Plusieurs d'entre eux ont exprimé, lors des discussions de groupes, leur incompréhension concer- 
nant l'attirance de certains jeunes pour la vie de gang. En plus d'y voir une perte de temps, plusieurs pensaient que ce style de vie tend à être dangereux.

La perception qu'ont les jeunes participants de l'étude à propos des gangs et de la violence des gangs les incite à limiter leurs interactions avec ce milieu, le percevant comme une source d'inquiétude pour leur sécurité. L'évitement actif leur a donc permis de s'éloigner de l'influence violente des gangs (Kelly et al., 2009; Lopez, Wishard, Gallimore et Riviera, 2006; Meyer et Astor, 2002). Résultat de la présence des gangs et de leur influence dans leur vie à l'école et dans leur quartier, de nombreux adolescents craignent maintenant ces groupes (Meyer et Astor, 2002). Ces jeunes sont persuadés qu'en évitant le contact avec les gangs, ils s'épargnent du même coup des situations dangereuses dans leur quartier. Toutefois, l'évitement et le fait de rester le plus possible à l'intérieur pourraient contribuer à réduire de saines habitudes de vie.

Même si l'évitement actif est un moyen efficace de se soustraire à l'influence des gangs, ce recours peut nuire à la vie sociale des adolescents (Howard, Kaljee et Jackson, 2002). Ces derniers doivent donc mener une vie sociale ailleurs, dans le cadre des activités organisées par les centres communautaires, par exemple. Sans une telle option, les jeunes passeraient leur temps chez eux lorsqu'ils ne sont pas à l'école. Les rares possibilités de socialisation sont, pour ces adolescents en croissance, autant de chances manquées de s'épanouir grâce à des activités saines menées dans un environnement sécuritaire (Shields, Nadasen et Pierce, 2008).

Les jeunes ont en général présenté des perceptions variées à l'égard des gangs et des violences associées à leur milieu. Comprendre leur point de vue pourrait aider à concevoir des programmes d'intervention auprès des adolescents à risque de se joindre à des gangs ainsi qu'à les informer et les influencer de manière positive lorsqu'ils doivent faire face à des choix difficiles.

Selon les jeunes interrogés, c'est parce qu'ils cherchent à être acceptés et qu'ils ont besoin d'un soutien qu'ils ne reçoivent pas dans leur famille que certains jeunes se joignent à des gangs. Et faire partie d'un gang contribuerait à créer un sentiment d'appartenance et à leur offrir ce qu'ils recherchent. Les travaux de Kelly, Anderson, Hall, Peden et Cerel (2011) ont permis de relever la variabilité des raisons évoquées par les jeunes qui les inciteraient à faire partie d'un gang, l'une d'elles étant qu'ils y sont acceptés (Kelly et al., 2011). L'idée voulant que les 
membres des gangs y cherchent à combler un vide n'est d'ailleurs pas nouvelle (Thornberry et al., 2003; Swetnam et Pope, 2001). D'autres recherches ont montré que l'atmosphère «familiale» trouvée au sein des gangs est leur principal attrait (Decker et Van Winkle, 1996). Les membres de gang s'apporteraient, les uns aux autres, soutien et protection (Maxson et Whitlock, 2002; Maxson, Whitlock et Klein, 1998; Thornberry et al., 2003). Ces jeunes croient donc fermement que ceux dont la vie familiale laisse à désirer se tournent vers les membres de leur gang pour du soutien.

Malgré que les adolescents à risque pensent qu'un gang peut contribuer à développer un sentiment d'appartenance entre les membres du groupe et à offrir le soutien qui manque dans leur famille, ils pensent tout de même que le prix à payer pour en faire partie demeure élevé. En effet, les travaux de Kelly et al. (2011) indiquent que les jeunes non membres sont d'avis que leurs pairs à risque doivent adopter des comportements dangereux pour entrer dans des gangs et cette impression est partagée par plusieurs jeunes de la présente étude. Un bon nombre d'entre eux ont en effet évoqué des situations dans lesquelles leurs pairs ont eu recours à la violence pour entrer dans un gang; et, selon eux, leurs camarades n'ont pas eu le choix. En fait, ces jeunes ont sans doute raison: les adolescents à risque ont probablement l'impression d'être à court d'options lorsqu'ils cherchent un environnement dans lequel ils se sentiront soutenus.

De plus, selon certains jeunes, ceux qui veulent entrer dans les gangs doivent parfois subir eux-mêmes des actes violents, comme c'est le cas des victimes du "passage à tabac». Des études ont en effet pu montrer que l'agression physique par un des membres est un rite de passage commun (Kelly et al., 2009). Ce serait en fait l'une des méthodes d'initiation les plus utilisées par les gangs, ce qui justifie que les jeunes ont ainsi l'impression que la violence et le risque font partie intégrante de la vie de gang. Mais selon Klein et Maxson (2006), de telles violences ne représenteraient qu'une infime partie du quotidien des membres. Tout de même, qu'importe la raison derrière les actes violents, les jeunes ont la nette impression que leurs pairs membres de gang finissent inévitablement par commettre des actes ayant des répercussions néfastes sur leur vie.

En fin de compte, ces adolescents d'horizons différents, qu'ils vivent en logements sociaux ou en familles monoparentales, font partie d'un milieu qui nuit à l'établissement d'une vie sociale saine. Considérant le 
milieu dont ils sont issus, il est naturel de se demander ce qui, au juste, a forgé leur perception des gangs et pourquoi ils n'ont pas succombé à l'appel de ce style de vie. Ont-ils été influencés par ce qu'ils voient dans leur quartier? Ou par le fait d'avoir un parent en prison? Comment crée-t-on enfin une relation parent-enfant au sein d'une famille qui soit assez forte pour fournir un environnement stable, rendant ainsi la vie de gang moins reluisante aux yeux d'un jeune? Ces adolescents semblent en effet comprendre les risques de la vie de gang et que, malgré ses charmes occasionnels, elle apporte parfois plus de mal que de bien. Leur expérience de vie et leurs interactions sociales passées semblent en tout cas avoir eu une influence très positive dans leur vie.

\section{Limites de la recherche}

La présente recherche comporte quelques limites. Dans les groupes de discussion, de composition hétérogène, il se peut que les participants se soient influencés les uns les autres ou n'aient pas osé émettre leurs vraies opinions. Il a été toutefois estimé que la présence de jeunes issus du même milieu les aurait encouragés à s'ouvrir davantage. Les différences culturelles entre la chercheure et les adolescents sont un autre facteur ayant pu influencer les réponses des jeunes, même si l'assistant ayant aidé à la collecte de données était issu d'un milieu culturel semblable à celui des jeunes. Il demeure que la présente étude éclaircit la question du point de vue des jeunes non membres de gangs sur leurs pairs qui se joignent aux gangs.

\section{Conclusion}

Comme mentionné plus haut, l'environnement des adolescents exerce sur eux une influence importante (Bronfenbrenner, 1979). La présente étude montre que les gangs et la violence qui y est liée agissent de manière directe et indirecte sur les jeunes à risque, en plus de fournir des éléments pouvant jouer un rôle dans le développement physique et mental de ces jeunes. Comprendre comment les adolescents à risque perçoivent les gangs et la violence des gangs pourrait permettre aux spécialistes de trouver de nouvelles manières d'intervenir auprès de ces jeunes. 


\section{Références}

Bronfenbrenner, U. (1979). The ecology of human development: Experiments by nature and design. Cambridge, MA: Harvard University Press.

Creswell, J. W. (1998). Qualitative inquiry and research design: Choosing among five traditions. Thousand Oaks, CA: Sage Publications.

Decker, S. et Van Winkle, B. (1996) Life in the gang. New York, NY: Cambridge University Press.

Dishion, T. J., Nelson, S. E. et Yasui, M. (2005). Predicting early adolescent gang involvement from middle school adaptation. Journal of Clinical Child and Adolescent Psychology, 34, 62-73.

Howard, D. E., Kaljee, L. et Jackson, L. (2002). Urban African American adolescents'perceptions of community violence. American Journal of Health Behavior, 26(1), 56-67.

Kelly, S. et Anderson, D. (2012). Adolescents, Gangs, and Perceptions of Safety, Parental Engagement, and Peer Pressure. Journal of Psychosocial Nursing and Mental Health Services. 50(10). 20-28.

Kelly, S., Anderson, D. et Peden, A. (2009). The psychological effect of exposure to gang violence on youth: A pilot study. Journal of Gang Research, 16(4), 35-52.

Kelly, S., Anderson, D., Hall, L., Peden, A. et Cerel, J. (2011). Adolescent males' perceptions of gangs and gang violence. Journal of Gang Research, 19(1), 1-8.

Klein, M. W. et Maxson, C. L. (2006). Street Gang Patterns and Policies. New York, NY: Oxford University Press.

Krueger, R. A. et Casey, M. A. (2000). Focus Groups. A Practical Guide for Applied Research $3^{\text {rd }}$ edition. Thousand Oaks, CA: Sage Publications.

Lopez, E. M., Wishard, A., Gallimore, R. et Riviera, W. (2006). Latino high school students' perceptions of gangs and crews. Journal of Adolescent Research, 21(3), 299-318.

Maxson, C. L. et Whitlock, M. L. (2002). Joining the Gang: Gender Differences in Risk Factors for Gang Membership. Dans C. R. Huff (dir.), Gangs in America $3^{\text {rd }}$ edition (p. 19-36). Thousand Oaks, CA: Sage Publications.

Maxson, C. L., Whitlock, M. L. et Klein, M. W. (1998). Vulnerability to street gang membership: Implications for practice. Social Service Review, 70-91.

Meyer, H. A. et Astor, R. A. (2002). Child and parent perspectives on routes to and from school in high crime neighborhoods. Journal of School Violence, 1(4), 101-128.

Shields, N., Nadasen, K. et Pierce, L. (2008). The effects of community violence on children in Cap Town, South Africa. Journal of Child Abuse and Neglect, 32(5), 589-601.

Swetnam, J. et Pope, J. (2001). Gangs and gang activity in a non-metropolitan community: The perceptions of students, teachers, and police officers. Social Behavior and Personality, 29(2), 197-207. 
Thornberry, T. P., Krohn, M. D., Lizotte, A. J., Smith, C. A. et Tobin, K. (2003). Gangs and delinquency in developmental perspective. New York, NY: Cambridge University Press.

Walker-Barnes, C. J. et Mason, C. A. (2001). Ethnic differences in the effect of parenting on gang involvement and gang delinquency: A longitudinal, hierarchical, linear modeling perspective. Child Development, 72(6) 18191831.

\title{
Gangs and Gang Violence: What Non-Gang Member Adolescents Think about Gangs and Gang Violence in their Neighborhood
}

\begin{abstract}
Gang violence is a growing societal problem. The purpose of this study was to explore the perceptions of non-gang member adolescents about gangs, gang violence, and the influence they believe gang violence has on their community. Twenty adolescents participated in focus groups. Four themes about gangs emerged from semistructured interviews: 1) a sense of belonging, 2) a price to be paid, 3) I just don't get it, and 4) active avoidance. These adolescents had different perceptions of gangs and gang violence. Understanding their views can lay the foundation for developing public health programs aimed at educating them about gangs and the consequences of gang involvement.
\end{abstract}

KEYWORDS • Youth, gangs, gang violence, neighborhood, community.

\section{Las pandillas callejeras y la violencia en sus barrios. Percepciones de jóvenes que no son miembros de las pandillas}

RESUMEN - La violencia de las pandillas es un problema de sociedad en pleno crecimiento. El presente estudio tiene como objetivo abordar la percepción que los adolescentes que no son miembros de pandillas tienen sobre éstas, sobre la violencia con las cuales las pandillas están asociadas y sobre la influencia que, según ellos, la violencia ejerce en su barrio. Veinte adolescentes han participado en los grupos de discusión y en entrevistas semi-dirigidas, lo que permitió extraer cuatro temáticas principales: 1) un sentimiento de pertenencia, 2) un precio a pagar, 3) la incomprehénsion 4) la evitación activa. Las percepciones sobre las pandillas y la violencia difieren de un adolescente a otro. Comprender sus puntos de vista, podría ayudar a la concepción de programas de salud pública que apunten a educar a los jóvenes con respecto a las pandillas callejeras y a las consecuencias de una eventual participación en tales grupos.

palabras CLAVE - Jóvenes, pandillas callejeras, violencia de las pandillas, barrio, comunidad. 